\title{
Classical generalized constant coupling model for geometrically frustrated antiferromagnets
}

\author{
Angel J. García-Adeva用 and David L. Huber \\ Department of Physics; University of Wisconsin-Madison; Madison, WI 53706
}

\begin{abstract}
A generalized constant coupling approximation for classical geometrically frustrated antiferromagnets is presented. Starting from a frustrated unit we introduce the interactions with the surrounding units in terms of an internal effective field which is fixed by a self consistency condition. Results for the magnetic susceptibility and specific heat are compared with Monte Carlo data for the classical Heisenberg model for the pyrochlore and kagomé lattices. The predictions for the susceptibility are found to be essentially exact, and the corresponding predictions for the specific heat are found to be in very good agreement with the Monte Carlo results.
\end{abstract}

PACS numbers: 75.10.Hk, 75.30.Cr, 75.40.Cx 


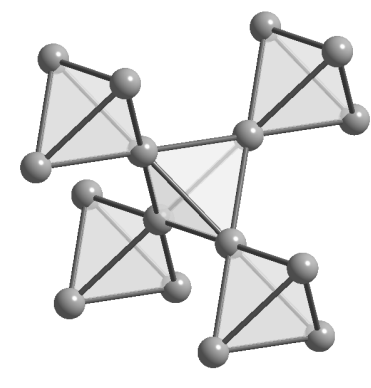

(a) Pyrochlore lattice.

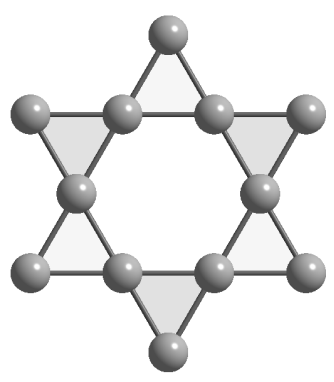

(b) Kagomé

lattice.

FIG. 1: The magnetic lattices considered in this work.

Introduction.- In the last several years, geometrically frustrated antiferromagnets (GFAF) have emerged as a new class of magnetic materials with uncommon physical properties and have received a vast amount of attention (see [1] and references therein). In these materials, the elementary unit of the magnetic structure is the triangle, which makes it impossible to satisfy all the antiferromagnetic bonds at the same time, with the result of a macroscopically degenerated ground state. Examples of GFAF are the pyrochlore and the kagomé lattices. In the former, the magnetic ions occupy the corners of a 3D arrangement of corner sharing tetrahedra; in the later, the magnetic ions occupy the corners of a 2D arrangement of corner sharing triangles (see Fig. 1). In the case of materials which crystallize in the pyrochlore structure, the magnetic susceptibility follows the Curie-Weiss law down to temperatures well below the Curie-Weiss temperature. At this point, usually of two orders of magnitude smaller than the Neél point predicted by the standard mean field (MF) theory, some systems exhibit some kind of long range order (LRO), whereas others experience a transition to a spin glass state (SG). This is a striking feature for a system with only a marginal amount of disorder. Finally, there some pyrochlores which do not exhibit any form of order whatsoever, and are usually regarded as spin liquids. In the case of the kagomé lattice, even though there are very few real systems where this structure is realized, the magnetic properties fall in two great categories: the vast majority of the compounds studied show a transition to a LRO state with non collinear configuration of spins, and a few systems exhibit no LRO, but a SG like transition.

For these reasons, it is easy to understand the large amount of attention, both from the experimental and the theoretical point of view, these systems have attracted. From the theoretical point 
of view, a number of techniques have been used to try to understand the origin of the puzzling properties mentioned above. All the theoretical results seem to indicate that for these geometries the classical Heisenberg model with only nearest neighbor interactions does not order at any finite temperature, in agreement with Monte Carlo (MC) results [2, 3, , , , 5, 6, 7]. There are also a relatively few works which have dealt with the quantum effects in these systems [8]. However, the main interest during last years has been in the classical GFAF.

In any case, the current theories have to deal with two main problems: the first one is that in real systems there are additional effects, which can be dipole-dipole interactions, small anisotropies, or further neighbor interactions, which are not easy to include in the theory, and so make it very difficult to compare with experimental results. However, this fact can be circumvented by using MC data calculated for the particular assumptions of the model. The second problem is that these models only provide qualitative agreement even when compared with MC data. In this sense, the single unit approximation, is the model which probably gives the best quantitative agreement when compared with susceptibility MC data for the pyrochlore lattice [2]. However, in order to reach such a good agreement, it is necessary to introduce an ad hoc rescaling procedure with an unclear physical justification. In the other hand, the infinite component spin vector model provides qualitative agreement for the kagomé case [7].

Obviously, the difficulty to find reliable models is rooted in the complexity of the geometry of these systems. Therefore, we think that, before embarking on the development of complicated models (which receive small support even from MC data obtained for the particular assumptions of the model), we should try to find simpler reliable approximation schemes which, as a first step, are in good qualitative and quantitative agreement with MC data. At the same time, these models should be as simple as possible (mathematically speaking), in order to be easily generalized to include additional interactions that play an important role in real systems. Only in this way, can we compare the models with data obtained from real systems, without this comparison being obscured by the complexity of the mathematical formalism.

In this work, we deal with the first of the two steps mentioned in the previous paragraph. We introduce a generalization of the well known constant coupling (CC) method [9], which has been successfully applied to the study of 3D standard ferro and antiferromagnets. Our method, which we will call generalized constant coupling (GCC) method, takes into account the geometrical peculiarities of the frustrated structures mentioned above. In spite of its mathematical simplicity, the susceptibilities and specific heat calculated for the pyrochlore and kagomé lattices, in the para- 
magnetic region, are in excellent agreement with MC data for the classical Heisenberg model in these systems.

It is important to note that, even though we have focused the discussion of the present work in the context of geometrical frustration in Heisenberg systems, this approach represents, in fact, a general technique for the investigation of the thermodynamic properties of spin Hamiltonians in frustrated geometries and, thus, its applicability is not limited to the GFAF, but could be useful in the investigation of other physical systems where geometrical frustration is relevant (see ref. 1 for a discussion of other problems where geometrical frustration is relevant).

The model.- The Heisenberg model with only nearest neighbor (NN) interactions in the presence of a magnetic applied field $H_{0}$ is described by the Hamiltonian [10]

$$
H=J \sum_{\langle i, j\rangle} \vec{s}_{i} \cdot \vec{s}_{j}-H_{0} \sum_{i} s_{z_{i}}
$$

where $J$ is the positive antiferromagnetic coupling, $\vec{s}_{i}$ and $\vec{s}_{j}$ represent classical spins of modulus $s_{0}$ located in a pyrochlore or kagomé lattice and $s_{z_{i}}$ the corresponding component along the applied field, and the sum is done over pairs of NN.

The idea of our approximate method is based on the experimental fact that the spin-spin correlations of the classical Heisenberg in the pyrochlore lattice are always short ranged [2]. Therefore, it is a reasonable approximation to start by considering isolated units (tetrahedra or triangles, for the pyrochlore and kagomé lattices, respectively), and later add the interactions with the surrounding units by in an approximate way (this is in contrast with the standard CC method, in which the elementary magnetic unit is taken as a pair of magnetic ions). Thus, it is important to first study the properties of the individual units. This task has been carried out by Moessner and Berlinsky [2], and the result obtained for the partition function of an isolated unit with $p$ spins in the absence of applied field is given by

$$
Z_{p}=\frac{(4 \pi)^{p+1}}{(4 \pi \beta \widetilde{J})^{3 / 2}} \int_{0}^{\infty} x^{2}\left(\frac{\sin x}{x}\right)^{p} \exp \left(-\frac{x^{2}}{2 \beta \widetilde{J}}\right) d x
$$

where $\beta=1 / T$ (we will use units of the Boltzmann constant $k_{B}$ throughout this work, so the energies are expressed in absolute temperature units), and we have introduced the effective coupling $\widetilde{J}=J s_{0}^{2}$. Expressions for units with 2, 3, and 4 ions can be found in the original work by those authors.

The susceptibility per spin, $\chi_{p}$, can be easily calculated from the partition function by using the 
fluctuation-dissipation theorem, and is given by

$$
\chi_{p}(\widetilde{T})=\frac{\left\langle S_{p}^{2}\right\rangle}{3 p T}=\frac{2 \widetilde{T}}{3 p J} \frac{\partial}{\partial \widetilde{T}} \ln Z_{p},
$$

where $\left\langle S_{p}^{2}\right\rangle$ represents the thermal average of the total spin of the unit with $p$ ions, and we have introduced the adimensional temperature $\widetilde{T}=\frac{T}{\widetilde{J}}$.

Next, we introduce the interaction with neighboring units as an unknown internal effective field, $H_{1}$, created by the $(p-1) \mathrm{NN}$ ions outside the unit. The $\mathrm{CC}$ approximation consists of taking this internal field as $H_{1}=(p-1) H^{\prime}$, where $H^{\prime}$ is the average internal field acting on a spin due to each of its NN. For example, in the case of the standard CW model, the internal effective field is given by $H_{1}=2(p-1) H^{\prime}$, as the ions are considered separately, and each has $2(p-1) \mathrm{NN}$ in the corner sharing structures considered in this work (see fig. 11). The internal field is evaluated by imposing the self consistent condition of equating the magnetization per spin in the field with that of a unit in the field, which can be mathematically stated as

$$
s_{0} L\left(s_{0}\left(H_{0}+2(p-1) H^{\prime}\right) / T\right)=\frac{m_{p}\left(H_{0}+(p-1) H^{\prime}\right)}{p},
$$

where the left side of the equation corresponds to the value of the magnetization per spin in the classical limit of the Curie-Weiss model, with $L(x)=\operatorname{coth} x-\frac{1}{x}$ the so called Langevin function [10]. The right side corresponds to the magnetization per spin of the isolated unit under the influence of the internal field. In the general case, equation (4) can only be solved numerically. However, in the paramagnetic regime [11], we can put, for small fields, $\left(H_{0}+H_{1}\right) / T \ll 1$,

$$
\frac{m_{p}\left(H_{0}+(p-1) H^{\prime}\right)}{p} \approx \chi_{p}(T)\left(H_{0}+(p-1) H^{\prime}\right)
$$

for the magnetization per spin in the cluster, where the susceptibility per spin is given by expression (3), and

$$
s_{0} L\left(s_{0}\left(H_{0}+2(p-1) H^{\prime}\right) / T\right) \approx \frac{s_{0}^{2}}{3 T}\left(H_{0}+2(p-1) H^{\prime}\right) .
$$

Taking into account eqs. (5) and (6), we can solve equation (4) very easily in terms of the function

$$
\varepsilon_{p}(\widetilde{T})=\frac{\left\langle S_{q}^{2}\right\rangle}{p s_{0}^{2}}-1=\frac{2 \widetilde{T}^{2}}{p} \frac{\partial}{\partial \widetilde{T}} \ln Z_{q}(\widetilde{T})-1,
$$

to give $H^{\prime}=\frac{\varepsilon_{p}(\widetilde{T})}{(p-1)\left[1-\varepsilon_{p}(\widetilde{T})\right]} H_{0}$, and the corresponding susceptibility per ion is then given by

$$
\chi_{p}^{g c c}(\widetilde{T})=\frac{1}{3 \widetilde{T}} \frac{1+\varepsilon_{p}(\widetilde{T})}{1-\varepsilon_{p}(\widetilde{T})} .
$$


Another interesting quantity we can readily evaluate in this model is the specific heat in zero applied field. In this limit, the internal field will be identically zero, and the specific heat will be identical to the specific heat calculated for non interacting units. Taking into account the relation $\left\langle\vec{s}_{i} \cdot \vec{s}_{j}\right\rangle=\frac{s_{0}^{2}}{p-1} \varepsilon_{p}(\widetilde{T})$ it can be easily shown that the specific heat per spin is given by

$$
C_{p}=\frac{\partial}{\partial \widetilde{T}} \varepsilon_{p}(\widetilde{T})
$$

Comparison with MC data. - Let us first consider the susceptibility obtained from the classical GCC model for the pyrochlore lattice. As can be seen from the observation of Fig. 2(a), the agreement between the theoretical curve and MC data [2] can be considered as exact in whole the temperature range. Moreover, at $T=0 \mathrm{~K}$, the value predicted by our model is $1 / 8 \widetilde{J}$, which is the value obtained from MC calculations. In the inset of that figure, we can see how the theory even predicts the kink in the susceptibility as one approaches very small temperatures.

In oder to corroborate that this surprising agreement is not a coincidence, we also compared the susceptibility calculated from the GCC model with MC data for the kagomé lattice [6], which can be seen in Fig. 2(b), Again, the curve predicted by our model can be considered as exact in whole the temperature range. Moreover, the value predicted by this model at $T=0 \mathrm{~K}$ is $1 / 6 \widetilde{J}$, again the exact value.

It is important to stress that the curves depicted in Figs. 2(a) and 2(b) have not been rescaled in any sense, nor contain any fit parameter. As far as we know, no other model is able to predict a such accurate susceptibility in the whole temperature range, considering both the pyrochlore and the kagomé lattice on an equal footing.

In order to further check the accuracy of the model, we decided to compare its predictions for the specific heat. The results are depicted in Fig. 3, together with MC data [4, 5] for these systems. Again, the agreement is excellent. For the pyrochlore lattice, the calculated specific heat is essentially exact down to $0 \mathrm{~K}$, where a value of $3 / 4$ is obtained [ $\beta$ ]. However, for the kagomé lattice, the exact value is $11 / 12$, whereas our model predicts a value of 1 . In any case, this deviation is expected to occur, as our model does not include the effect of zero modes, which are known to be especially important in the kagomé case, where they give rise to the phenomenon known as order by disorder [5], 8]. In contrast, MC data suggest that this mechanism is absent in the Heisenberg pyrochlore.

Conclusions. - In this work we have presented a generalization of the so called constant coupling approach for geometrically frustrated antiferromagnets with a pyrochlore or kagomé structure. 


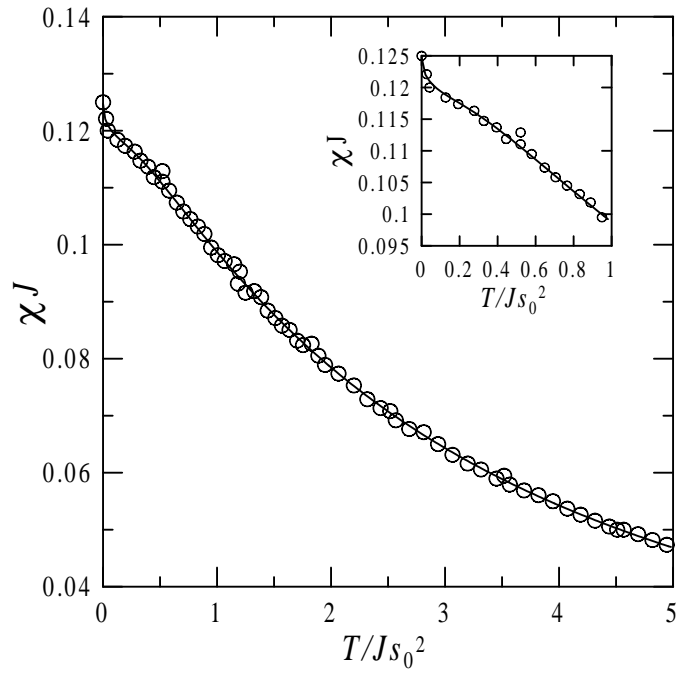

(a) Susceptibility of the pyrochlore lattice.

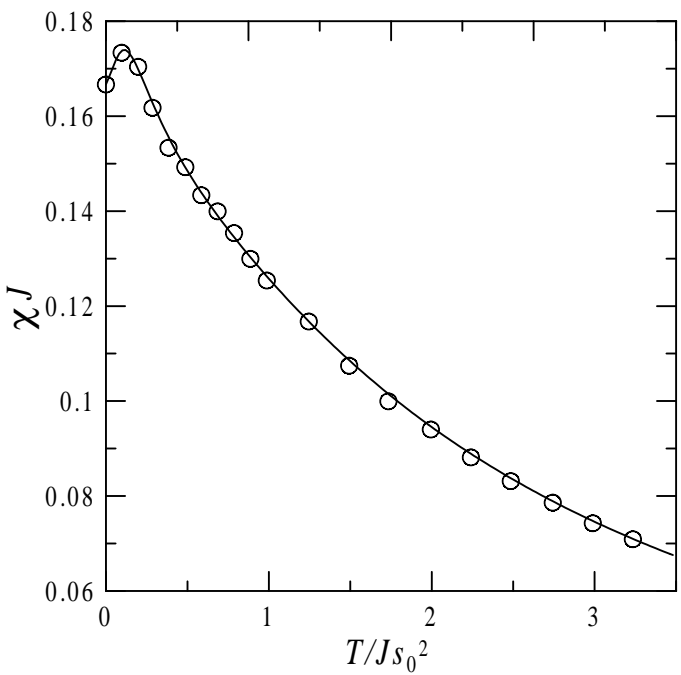

(b) Susceptibility of the Kagomé lattice.

The inset shows a detailed comparison at very low temperatures.

FIG. 2: Susceptibility from Monte Carlo calculations and the one predicted by the GCC model. Open circles are from MC simulations (obtained from [2] for the pyrochlore lattice and from [6] for the kagomé lattice). The solid line corresponds to the GCC model.

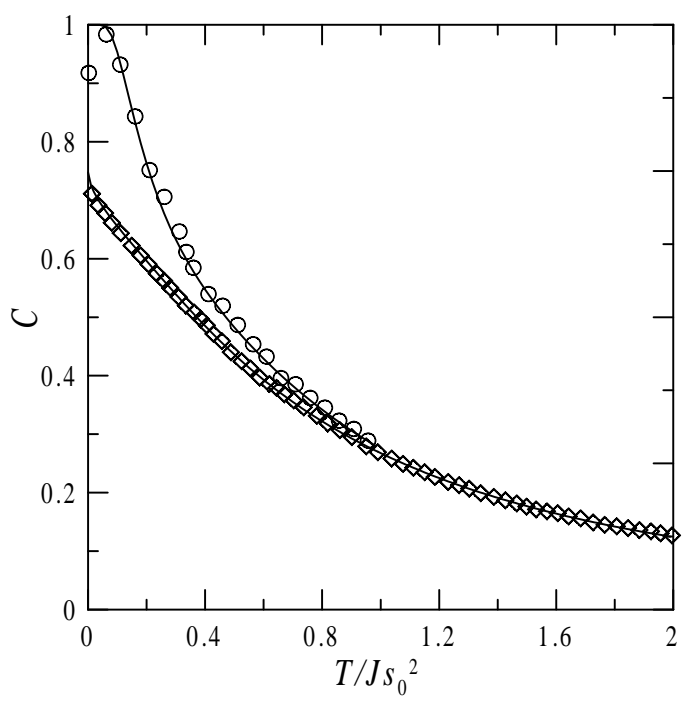

FIG. 3: Specific heat from Monte Carlo simulations and the one predicted by the GCC model: (open circles) Specific heat of the kagomé lattice [5]; (open diamonds) Specific heat of the pyrochlore lattice [⿰屯𠃌灬. The solid lines are the results of the GCC model. 
As a preliminary approach, we have considered the most simple description of these systems, which consists of a classical Heisenberg model with only nearest neighbors interactions. It seems clear from Monte Carlo results that, in this approximation, these systems remain paramagnetic in the whole temperature range. Analytical expressions for the static magnetic susceptibility and specific heat can be obtained in the framework of this model. These have been compared with Monte Carlo data for these systems. The predicted susceptibility is essentially exact in whole the temperature range for both types of lattice. The specific heat for the pyrochlore lattice is again found to be essentially exact down to zero temperature, whereas it deviates from the real value in the kagomé case, as our model does not include the so called order by disorder phenomenon, which is especially important in this system at very low temperatures. It is specially remarkable that the curves that are compared with Monte Carlo data do not contain any fit parameter, or any rescaling factor.

Obviously, this is only a first step in the understanding of the uncommon features present in frustrated geometries. There are still a lot of puzzling questions regarding the nature of the spin glass and spin liquids observed in these systems, which we do not think can be understood in the framework of the constant coupling theory.

In any case, we think that the present model provides an excellent starting point to understand open questions that remain even in the paramagnetic regime, as are, to cite some examples, the effects of small anisotropies, dipole-dipole interactions, or the effects of a small amount of dilution with non-magnetic impurities in the magnetic lattice. Furthermore, the corresponding quantum generalized constant coupling method provides some insight on how the quantum effects manifest themselves in the magnetic properties of these systems [12]. It is important to note again, that the applicability of the present technique is not limited to the study of Heisenberg systems, but is useful for the investigation of the physical properties of other systems where geometrical frustration is relevant.

Angel García Adeva wants to thank M.A. Cazalilla for very stimulating discussions. This work has been partially supported by the Spanish MEC, under the Subprograma General de Formación de Personal Investigador en el Extranjero.

* Electronic address: garcia@landau.physics.wisc.edu

[1] Proceedings of the Highly Frustrated Magnetism 2000 conference; A. P. Ramirez, Ann. Rev. Mater. 
Sci. 24, 453 (1994); P. Schiffer and A. P. Ramirez, Comments Condens. Mater. Phys. 18, 21 (1996).

[2] R. Moessner and A. J. Berlinsky, Phys. Rev. Lett. 83, 3293 (1999)

[3] R. Moessner and J. T. Chalker, Phys. Rev. Lett. 80, 2929 (1998); R. Moessner, Phys. Rev. B 57, R5587 (1998); R. Moessner and J. T. Chalker, Phys. Rev. B 58, 12049 (1998); J. N. Reimers, A. J. Berlinsky, and A. C. Shi, Phys. Rev. B 43, 865 (1991); A. B. Harris, C. Kallin, and A. J. Berlinsky, Phys. Rev. B 45, 2899 (1992).

[4] J. N. Reimers, Phys. Rev. B 45, 7287 (1992).

[5] J. T. Chalker, P. C. W. Holdsworth, and E. F. Shender, Phys. Rev. Lett. 68, 855 (1992).

[6] J. N. Reimers and A. J. Berlinsky, Phys. Rev. B 48, 9539 (1993).

[7] D. A. Garanin and B. Canals, Phys. Rev. B 59, 443 (1999).

[8] A. B. Harris, A. J. Berlinsky, and C. Bruder, J. Appl. Phys. 69, 5200 (1991); B. Canals and C. Lacroix, Phys. Rev. Lett. 80, 2933 (1998); Phys. Rev. B 61, 1149 (2000); M. Isoda and S. Mori, J. Phys. Soc. Jpn. 67, 4022 (1998); A. Chubukov, Phys. Rev. Lett. 69, 832 (1992); A. J. García and D. L. Huber, Phys. Rev. Lett. (to be published).

[9] P. W. Kastelejein and J. van Kranendonk, Physica 22, 317 (1956); N. Goveas and G. Mukhopadhyay, Phys. Scripta 56, 528 (1997).

[10] J. S. Smart, "Effective field theories of magnetism" (Saunders, Philadelphia, 1966).

[11] As commented in the introduction, the classical Heissenberg model with NN interactions remains paramagnetic down to $T=0 \mathrm{~K}$. Therefore, a series expansion of the magnetization is well justified in whole the temperature range, as long as the applied field is small.

[12] A. J. García and D. L. Huber, (unpublished). 